\title{
Cumulative risk assessment in unstable angina: clinical, electrocardiographic, autonomic, and biochemical markers
}

\author{
S Kennon, C P Price, P G Mills, P K MacCallum, J Cooper, J Hooper, H Clarke, \\ A D Timmis
}

See end of article for authors' affiliations

.....................

Correspondence to: Dr Simon Kennon, Cardiology Department, London Chest Hospital, Bonner Road, London E2 9JX, UK;

srok@dircon.co.uk

Accepted

2 October 2002

\section{METHODS \\ Patients}

Consecutive patients with non-ST elevation acute coronary syndromes were recruited if they fulfilled criteria for Braunwald class 3B unstable angina. ${ }^{16}$ Patients with serum creatine kinase $\mathrm{MB}(\mathrm{CKMB})$ concentrations $\geqslant 4.0 \mu \mathrm{g} / \mathrm{l}$ without Q wave development were diagnosed as non-Q wave myocardial infarction. Electrocardiographic changes (ST depression, T wave inversion) were not required for inclusion but patients who developed Q waves were excluded. Other exclusion criteria were myocardial infarction within the previous 21 days, angioplasty within the preceding six months, cardiac failure (New York Heart Association functional class III or IV), chronic severe illness, renal impairment (creatinine $>200 \mu \mathrm{mol} / \mathrm{l}$ ), oral anticoagulation, aortic stenosis, and any abnormality preventing interpretation of ST changes on Holter analysis (left bundle branch block, paced rhythm, digoxin, severe left ventricular hypertrophy). The study protocol was approved by the local (East London and The City) research ethics committee and informed consent was obtained from all subjects before recruitment

\section{Data collection}

Clinical data

Baseline clinical characteristics including demographic, clinical, and biochemical data were collected prospectively. Details of clinical history and inpatient management were recorded. Current smokers and those who had stopped smoking less than two weeks before presentation were classified as smokers. All others were grouped as non-smokers for this study.

Abbreviations: $A \cup C$, area under the receiver operating characteristic curve; $C A B G$, coronary artery bypass graft; $C K M B$ creatine kinase $M B$ fraction; ELISA, enzyme linked immunosorbent assay; FRISC II, Fragmin and fast revascularization during instability in coronary artery disease; PTCA, percutaneous transluminal coronary angioplasty; SDANN, standard deviation of 5 minute mean RR intervals; TIMI, thrombolysis in myocardial infarction 


\section{Electrocardiographic analysis}

Presenting ECGs recorded in the emergency department were analysed for evidence of ischaemia. ST depression was measured at $80 \mathrm{~ms}$ after the J point. Both ST depression and T wave inversion had to occur in at least two contiguous leads and to be at least $0.1 \mathrm{mV}$ to be considered significant.

\section{Blood sampling and biochemical analysis}

In addition to samples taken for routine laboratory analysis according to hospital protocols, samples were also taken for CKMB and troponin $\mathrm{T}$ analysis on admission (before antithrombotic treatment) and at 12,24, and 48 hours after admission. Serum CKMB concentrations were measured using a one step sandwich immunoassay (Bayer Immuno 1 Analyser, Bayer Plc, Newbury, UK). The lower detection limit was $0.1 \mu \mathrm{g} / \mathrm{l}$. The coefficient of variation was $6.1 \%$ at $4.6 \mu \mathrm{g} / \mathrm{l}$ and $5.8 \%$ at $15.3 \mu \mathrm{g} / \mathrm{l}$. The cut off for diagnosis of myocardial infarction was $4.0 \mu \mathrm{g} / \mathrm{l}$ (manufacturer's data sheet). Troponin $\mathrm{T}$ concentrations were determined by using an enzyme linked immunosorbent (ELISA) one step sandwich assay (EnzymunTest, Roche Diagnostics, Lewes, UK). The lower detection limit was $0.02 \mu \mathrm{g} / \mathrm{l}$ and the coefficient of variation was $9 \%$ at $0.35 \mu \mathrm{g} / \mathrm{l}$ and $3.2 \%$ at a concentration of $5.44 \mu \mathrm{g} / \mathrm{l}$ (manufacturer's data sheet). The cut off point used was $0.1 \mu \mathrm{g} / \mathrm{l}$.

\section{Holter monitoring}

ST segment and heart rate variability were monitored continuously for 48 hours within 24 hours of admission. ST segment shift and heart rate variability were determined as previously described. ${ }^{17}{ }^{18}$ Briefly, frequency modulated dual channel recorders (Marquette Series 8000, Delmar, Irvine, California, USA) were used and tapes were analysed, without knowledge of the clinical outcome, using a commercially available system (Delmar Accuplus 363). The recordings were analysed for both spectral and non-spectral measures of heart rate variability. The measures calculated were ratio of low $(0.04-0.15 \mathrm{~Hz})$ to high $(0.15-0.4 \mathrm{~Hz})$ frequency, mean of all coupling intervals between qualified beats, the standard deviation about the mean, proportion of adjacent RR intervals more than 50 ms different, root mean square of the difference of successive RR intervals, the standard deviation of five minute mean RR intervals (SDANN), and the mean of all five minute standard deviations of RR intervals (SD). Attending physicians were blinded to the results of 48 hour tape analysis and assays for serum CKMB and troponin $\mathrm{T}$.

\section{Follow up}

Patients were followed up for 12 months. The primary end points were death from cardiac causes and non-fatal myocardial infarction. Myocardial infarction was diagnosed if any two of the following criteria were fulfilled: (1) cardiac chest pain lasting at least 30 minutes; $(2) \geqslant 0.1 \mathrm{mV}$ ST elevation in at least one standard lead or $\geqslant 0.2 \mathrm{mV}$ ST elevation in two or more contiguous chest leads; or ( 3 ) CK $\geqslant 400$ IU/l (upper limit of reference range: $200 \mathrm{IU} / \mathrm{l}$ ) or $\mathrm{CKMB} \geqslant 4.0 \mu \mathrm{g} / \mathrm{l}$.

\section{Statistical analysis}

Results for continuous variables are presented as means and standard deviations, and two sample $t$ tests were used to compare those with and without events. For variables not normally distributed results are presented as medians and interquartile ranges and compared using the Mann-Whitney $\mathrm{U}$ test. Categorical variables were compared using $\chi^{2}$ or Fisher's exact test. Variables significant $(p<0.05)$ on univariate analysis were selected for testing in a stepwise multiple logistic regression model. We then looked at the effect of adding variables insignificant on univariate analysis to test whether any of these became important after adjustment. Results from the logistic regression were expressed as the odds of an event relative to a reference category for categorical variables and as the relative odds associated with a one standard deviation increase in continuous variables. Cumulative risk scores were calculated by incremental analysis of all independent predictors of ischaemic events (death, non-fatal myocardial infarction) using the selected characteristics weighted by the coefficients from the logistic regression model. Cut off points for this score were selected to give false positive rates of $5-10 \%$. The discriminative ability of the risk scores was assessed using the area under the receiver operating characteristic (AUC) curve. This index ranges from 0.5 for a test with no discriminative ability to 1.0 for a test that discriminates perfectly. The internal validity of the model generated from the entire cohort was assessed by cross validation, whereby the cohort was randomly divided into two halves, one used to develop the model and the other to determine the fit and discriminative ability.

\section{RESULTS}

\section{Discharge diagnosis and outcome}

\section{Discharge diagnosis}

The study population consisted of 304 consecutive patients admitted with non-ST elevation acute coronary syndromes. The discharge diagnosis was non-Q wave myocardial infarction in $92(30 \%)$ and unstable angina in $212(70 \%)$.

Outcome: death and myocardial infarction

Thirty day follow up was obtained in 303 patients $(99.7 \%)$, of whom one died and 14 had non-fatal myocardial infarction. Thirteen of these events, including the death, occurred before discharge. Twelve month follow up was obtained in 289 patients $(95.1 \%)$, of whom 7 died and 21 had non-fatal myocardial infarction. All deaths during follow up were attributable to cardiac causes.

\section{Outcome: major adverse cardiac events}

During the first 30 days' follow up an additional 39 patients underwent revascularisation procedures (23 percutaneous transluminal coronary angioplasty (PTCA) and 16 coronary artery bypass graft (CABG)). After one year, 67 patients had undergone revascularisation procedures (34 PTCA and 33 CABG). There were therefore 54 major adverse cardiac events (death, non-fatal myocardial infarction, PTCA, and CABG) after 30 days and 95 after one year.

\section{Univariate predictors of death and non-fatal myocardial infarction Clinical factors}

Ischaemic events occurred more commonly in patients who were older and had diabetes (table 1). These events were also more common when the hospital course was complicated by left ventricular failure or chest pain that failed to settle within 12 hours of admission.

\section{Electrocardiographic factors}

Ischaemic events occurred more commonly in patients with $\mathrm{T}$ wave inversion on the presenting ECG (table 2). ST change was also associated with ischaemic events, particularly when recorded during Holter monitoring.

\section{Autonomic factors}

Analysis of heart rate variability showed that time domain indices tended to be lower among patients who had ischaemic events; frequency domain indices were similar between the groups (table 2 ).

\section{Biochemical factors}

Admission cholesterol and creatinine concentrations were, on average, higher in patients who had ischaemic events during follow up (table 3). Admission and peak CKMB concentrations were also higher in these patients. Among patients with 
Table 1 Clinical univariate predictors of death or non-fatal myocardial infarction at 30 days and 12 months

\begin{tabular}{|c|c|c|c|c|c|c|}
\hline \multirow[b]{2}{*}{ Variable } & \multicolumn{3}{|l|}{30 day follow up } & \multicolumn{3}{|l|}{12 month follow up } \\
\hline & No event $(n=288)$ & Event $(n=15)$ & $\mathrm{p}$ Value & No event $(n=261)$ & Event $(n=28)$ & $\mathrm{p}$ Value \\
\hline Age (years) & $60.1(11.6)$ & $64.5(9.6)$ & 0.16 & $60.0(11.1)$ & $67.5(11.3)$ & 0.001 \\
\hline Male sex & $170(72.3)$ & $14(93.3)$ & 0.11 & 192 (73.9) & $23 \quad(82.1)$ & 0.34 \\
\hline Diabetes & $50(21.2)$ & $5(33.3)$ & 0.28 & 53 (20.3) & 11 (39.3) & 0.03 \\
\hline Hypertension & $107(45.3)$ & $8(53.3)$ & 0.55 & 116 (44.4) & $14 \quad(50.0)$ & 0.58 \\
\hline Smoking & $74(31.4)$ & $4(26.7)$ & 0.7 & 85 (32.6) & $6 \quad(21.4)$ & 0.23 \\
\hline Chest pain $>12$ hours* & $15(6.5)$ & $4(26.7)$ & 0.01 & $16 \quad(6.2)$ & $7 \quad(25.0)$ & 0.001 \\
\hline LVF† & $70(30.2)$ & 13 (86.7) & $<0.0001$ & $96 \quad(37.4)$ & 15 (53.6) & 0.10 \\
\hline Arrhythmias $\ddagger$ & $2(0.9)$ & $2(13.3)$ & 0.006 & $2(0.8)$ & 2 (7.1) & 0.03 \\
\hline
\end{tabular}

Data are numbers (percentages) except for age, which is mean (standard deviation). *Patients with new episodes of chest pain $>12$ hours after admission; †clinical evidence of left ventricular failure (LVF) during admission; †documented arrhythmia during admission.

Table 2 Electrocardiographic and autonomic univariate predictors of death or non-fatal myocardial infarction

\begin{tabular}{|c|c|c|c|c|c|c|c|}
\hline \multirow[b]{2}{*}{ Variable } & \multicolumn{3}{|l|}{30 day follow up } & \multicolumn{4}{|l|}{12 month follow up } \\
\hline & No event $(n=288)$ & Event $(n=15)$ & $\mathrm{p}$ Value & No event $(n=261)$ & Event & $(n=28)$ & $\mathrm{p}$ Value \\
\hline \multicolumn{8}{|l|}{ Admission ECG } \\
\hline T wave inversion & $142(49.3 \%)$ & $13(86.7 \%)$ & 0.005 & $124(47.5 \%)$ & 24 & $(85.7 \%)$ & 0.001 \\
\hline ST depression & $84(29.2 \%)$ & $4(26.7)$ & 0.84 & $71 \quad(27.2 \%)$ & 12 & $(42.9 \%)$ & 0.09 \\
\hline \multicolumn{8}{|l|}{ Holter monitoring } \\
\hline Any ST depression & $76(28.6 \%)$ & 9 (69.2\%) & 0.002 & $67 \quad(27.2)$ & 15 & $(65.2 \%)$ & $<0.0001$ \\
\hline Any ST elevation & $48(18.1 \%)$ & $9(69.2 \%)$ & $<0.0001$ & $43(17.5 \%)$ & 12 & $(52.2 \%)$ & $<0.0001$ \\
\hline Any ST shift & $106(39.9 \%)$ & $12(92.3 \%)$ & $<0.0001$ & $95 \quad(38.6 \%)$ & 19 & $(82.6 \%)$ & $<0.0001$ \\
\hline \multicolumn{8}{|l|}{ Heart rate variability } \\
\hline pNN50 (\%) & $9.8(3.4-17.7)$ & $9.1(5.2-12.8)$ & 0.92 & $8.1(3.4-17.6)$ & 10.8 & $(5.9-12.8)$ & 0.46 \\
\hline SDNN (ms) & $113.3(32.1)$ & $93.7(17.0)$ & 0.03 & $113.1(33.3)$ & 98.8 & $(26.4)$ & 0.05 \\
\hline Mean NN (ms) & $910.9(158.1)$ & 808.0 (122.9) & 0.03 & $912.4(156.3)$ & 804.7 & (138.3) & 0.002 \\
\hline RMSSD (ms) & $41.0(22.3)$ & 42.0 (19.3) & 0.87 & $40.0(22.6)$ & 45.8 & $(26.4)$ & 0.27 \\
\hline $\mathrm{SD}(\mathrm{ms})$ & 41.7 (17.9) & $37.7(9.8)$ & 0.39 & $41.1(18.4)$ & 38.4 & $(15.2)$ & 0.49 \\
\hline SDANN (ms) & $100.8(28.7)$ & $81.9(14.8)$ & 0.02 & $100.8(29.5)$ & 85.7 & $(21.7)$ & 0.018 \\
\hline LF:HF & $1.67(0.98-3.52)$ & $2.59(1.11-6.44)$ & 0.47 & $1.9(0.99-3.56)$ & 1.36 & $6(1.08-2.77)$ & 0.31 \\
\hline
\end{tabular}

LF:HF, low frequency to high frequency ratio; mean NN, mean of all coupling intervals between qualified beats; pNN50, proportion of adjacent RR intervals more than 50 ms different; RMSSD, root mean square of the difference of successive RR intervals; SD, mean of all 5 minute standard deviations of RR intervals; SDANN, standard deviation of 5 minute mean RR intervals; SDNN, standard deviation about the mean.

Data for pNN50 and LF:HF are median (interquartile range) and for SDNN, mean NN, RMSSD, SD, and SDANN are mean (standard deviation).

Table 3 Biochemical univariate predictors of death or non-fatal myocardial infarction at 30 days

\begin{tabular}{|c|c|c|c|c|c|c|}
\hline \multirow[b]{2}{*}{ Variable } & \multicolumn{3}{|l|}{30 day follow up } & \multicolumn{3}{|l|}{12 month follow up } \\
\hline & No event $(n=288)$ & Event $(n=15)$ & $\mathrm{p}$ Value & No event $(n=261)$ & Event $(n=28)$ & $\mathrm{p}$ Value \\
\hline Cholesterol (mmol/l) & $5.66(1.04)$ & $6.11(1.34)$ & 0.1 & $5.62(1.12)$ & $6.11(1.22)$ & 0.05 \\
\hline Creatinine $(\mu \mathrm{mol} / \mathrm{l})$ & $102.8(21.6)$ & $118.2(25.8)$ & 0.015 & $103.2(22.6)$ & 113.9 (23.9) & 0.03 \\
\hline Glucose (mmol/l) & $6.4(5.7-8.3)$ & $7.2(6.5-13.0)$ & 0.02 & $6.5(5.7-8.2)$ & $6.6(6.0-13.0)$ & 0.17 \\
\hline Admission CKMB ( $\mu \mathrm{g} / \mathrm{l})$ & $1.5(0.8-3)$ & $6.7(4.4-9)$ & 0.0003 & $1.4(0.8-2.9)$ & $4.6(1.8-9)$ & $<0.0001$ \\
\hline Peak CKMB $(\mu \mathrm{g} / \mathrm{I})$ & $1.6(0.9-5.3)$ & $36.1(8.3-79.2)$ & $<0.0001$ & $1.6(0.9-4.2)$ & $15.9(3.7-64.8)$ & $<0.0001$ \\
\hline Troponin $T>0.1 \mu \mathrm{g} / \mathrm{l}$ at: & & & & & & $<0.0001$ \\
\hline 0 hours & $47(17.4 \%)$ & $8(57.1 \%)$ & $<0.0001$ & (15.9\%) & $(53.9 \%)$ & $<0.0001$ \\
\hline 12 hours & $63(24.3 \%)$ & $11(78.6 \%)$ & $<0.0001$ & $52 \quad(22.3 \%)$ & $(69.2 \%)$ & $<0.0001$ \\
\hline 24 hours & $64(24.8 \%)$ & $11(78.6 \%)$ & $<0.0001$ & $55 \quad(23.7 \%)$ & $17 \quad(65.4 \%)$ & $<0.0001$ \\
\hline 48 hours & $45(21.5 \%)$ & $10(90.9 \%)$ & $<0.0001$ & $(20.5 \%)$ & $(70.6 \%)$ & $<0.0001$ \\
\hline Any time & $78(27.8 \%)$ & $13(86.7 \%)$ & $<0.0001$ & $64 \quad(25.2 \%)$ & (78.6\%) & $<0.0001$ \\
\hline
\end{tabular}

Data for cholesterol, creatinine, and CKMB are mean (SD) and for glucose are median (interquartile range).

ischaemic events, the proportion with increased $(>0.1 \mu \mathrm{g} / \mathrm{l})$ troponin $\mathrm{T}$ concentrations at each sampling point and at any sampling point was over twice that of patients without events.

\section{Multivariate predictors of death and non-fatal myocardial infarction}

Thirty days

Clinical, electrocardiographic, autonomic, and biochemical factors were all independently predictive of ischaemic events during the first 30 days (table 4). Thus, in patients with Holter ST elevation or chest pain that failed to settle within 12 hours of admission, the odds of having an ischaemic event were increased 10.6 and 15.1 times, respectively. Any increase of troponin $\mathrm{T}>0.1 \mu \mathrm{g} / \mathrm{l}$ increased the odds of an event more than 30 times. Conversely, preservation of heart rate variability reduced the odds of an ischaemic event by $65 \%$ for every standard deviation (approximately $30 \mathrm{~ms}$ ) increase in SDANN.

Twelve months

In patients with $\mathrm{T}$ wave inversion on the presenting ECG or Holter ST elevation or depression, the odds of an ischaemic event during the first 12 months were increased 5.8, 4.4, and 3.2 times, respectively (table 4). Any increase of troponin $\mathrm{T}$ 
Table 4 Multivariate predictors of death or non-fatal myocardial infarction at 30 days and 12 months in 304 patients with non-ST elevation acute coronary syndromes

\begin{tabular}{lll}
\hline Variable & Odds ratio $(95 \% \mathrm{Cl})$ & $\mathrm{p}$ Value \\
\hline At 30 days & $32.53(3.59$ to 294.72$)$ & 0.002 \\
$\quad$ Troponin Tmax & $15.1(2.47$ to 91.21$)$ & 0.003 \\
$\quad$ Chest pain & $10.63(2.14$ to 52.8$)$ & 0.004 \\
$\quad$ Holter ST elevation & $0.35(0.14$ to 0.86$)$ & 0.022 \\
SDANN & $6.33(1.9$ to 21.09$)$ & 0.003 \\
At 12 months & $5.75(1.48$ to 22.25$)$ & 0.011 \\
$\quad$ Troponin Tmax* & $4.41(1.51$ to 12.92$)$ & 0.007 \\
T wave inversion & $3.24(1.11$ to 9.43$)$ & 0.031 \\
Holter ST elevation & $0.53(0.29$ to 0.96$)$ & 0.035 \\
$\quad$ Holter ST depression & & \\
SDANN & & \\
\hline *Troponin T >0.1 $\mu \mathrm{g} / \mathrm{l}$ at any time. &
\end{tabular}

$>0.1 \mu \mathrm{g} / \mathrm{l}$ increased the odds of an event more than six times. Preservation of heart rate variability reduced the odds of an ischaemic event by $47 \%$ for every standard deviation increase in SDANN.

\section{Predictive accuracy of risk markers for ischaemic events Thirty days}

Analysis of individual multivariate risk markers showed that positive predictive accuracies ranged from $8.7 \%$ for every standard deviation increase in SDANN to $15.8 \%$ for Holter ST elevation (table 5). Negative predictive accuracies (proportions of patients with negative predictors who did not sustain events) were consistently $>90 \%$. However, incorporation of all multivariate risk markers permitted calculation of a cumulative risk score (table 6) with substantial improvement in predictive accuracy. For scores $\geqslant 3.75$ the positive predictive accuracy for ischaemic death or non-fatal myocardial infarction in the first 30 days was $40 \%$. Scores $<3.75$ were associated with a negative predictive accuracy of $98.6 \%$. When internal validity was tested the AUC was high in both development $(\mathrm{AUC}=0.95)$ and test $(\mathrm{AUC}=0.95)$ models.

Twelve months

Positive predictive accuracies of individual multivariate risk markers for events in the first year ranged from $11.5 \%$ for

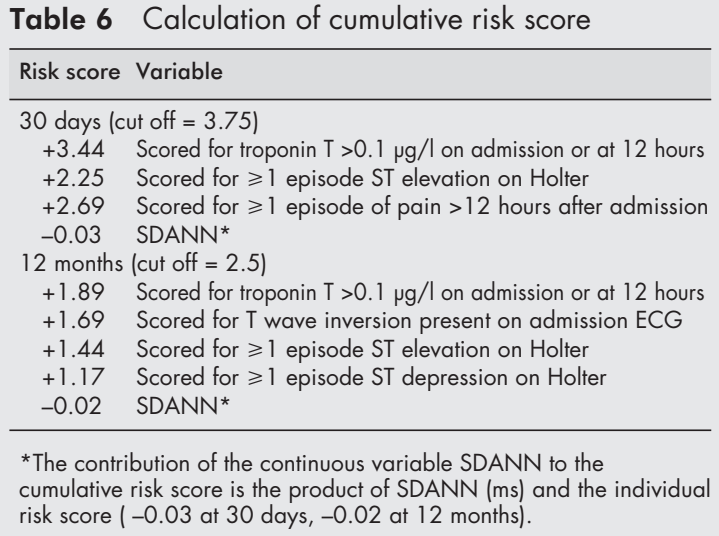

*The contribution of the continuous variable SDANN to the cumulative risk score is the product of SDANN (ms) and the individual risk score ( -0.03 at 30 days, -0.02 at 12 months).

every standard deviation increase in SDANN to $25.6 \%$ for troponin $\mathrm{T}>0.1 \mu \mathrm{g} / \mathrm{l}$ ( table 5 ). Again, the calculated cumulative risk score (table 6) improved predictive accuracy. For patients with risk scores $\geqslant 2.50$, the positive predictive accuracy for ischaemic death or non-fatal myocardial infarction in the first 12 months was $46.9 \%$. Scores $<2.50$ were associated with a negative predictive accuracy of $96.9 \%$. Predictive accuracy was slightly reduced in the test model $($ AUC $=0.84)$ compared with the development model ( $\mathrm{AUC}=0.93)$.

\section{Excluding data derived from continuous ECG (Holter) monitoring}

The analysis was repeated after ST segment shift and heart rate variability data were excluded. The calculated cumulative risk score retained true positive rates and negative predictive values of around $70 \%$ and $95 \%$, respectively, but positive predictive values at both 30 days and 12 months fell to $31.4 \%$ and $31.3 \%$, respectively.

\section{DISCUSSION}

This prospective cohort study has identified a range of clinical, electrocardiographic, autonomic, and biochemical factors all independently predictive of outcome in patients with non-ST elevation coronary syndromes. However, most risk markers had a low concentration of predictive accuracy when applied independently, potentially limiting their clinical value. For example, troponin $\mathrm{T}$, the best independent predictor of risk,

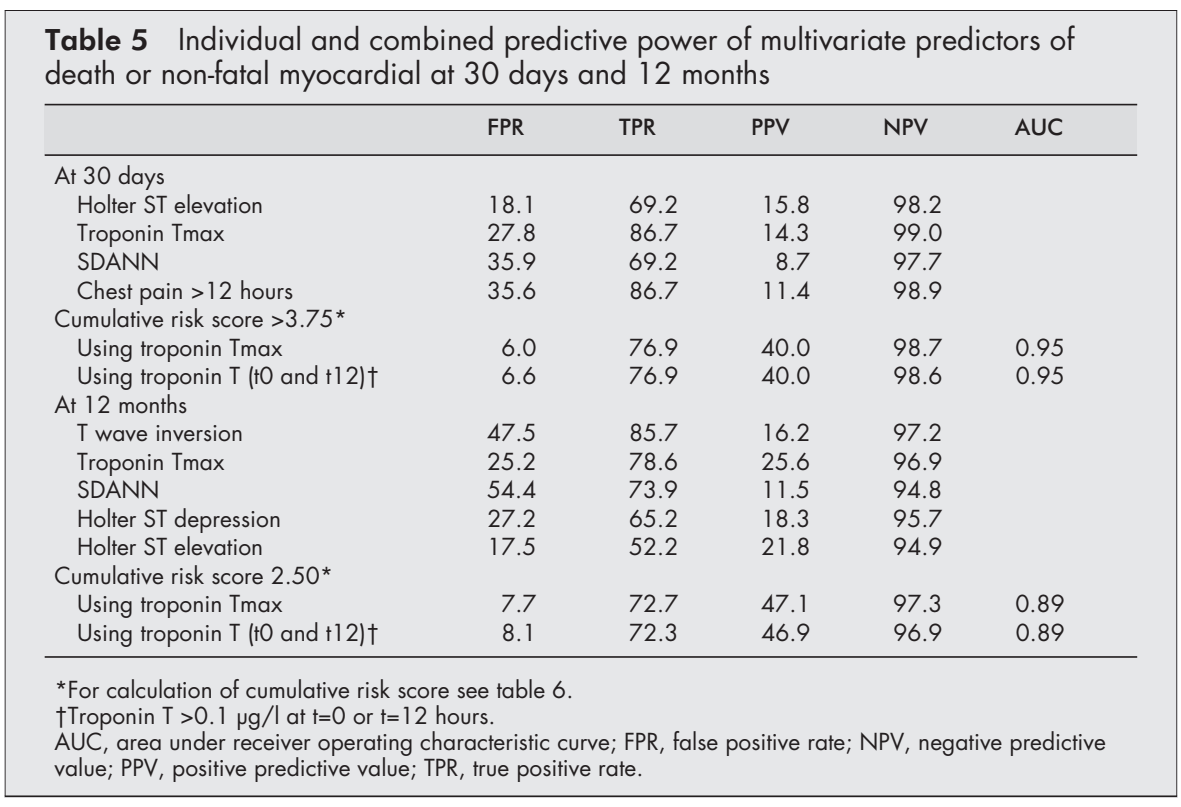


identified $>75 \%$ of patients who had events in the first 12 months, but $75 \%$ of those with increased concentrations had no event. Previous investigators have confirmed that troponins provide prognostic information independent of the resting ECG, ${ }^{12}$ but this study has extended these observations by showing that cumulative analysis of multiple independent determinants of risk, including Holter ST and heart rate variability data, can substantially improve predictive accuracy. This is of particular clinical relevance because it was applied to unselected patients with non-ST elevation acute coronary syndromes and no attempt was made to preselect high risk subgroups.

\section{Troponin T}

The minimal myocardial damage that may occur following transient or subocclusive thrombus formation in non-ST elevation coronary syndromes is now recognised as a major predictor of subsequent ischaemic events. In the present study, troponin $\mathrm{T}$ was the most powerful predictor of risk; however, its usefulness for risk stratification was limited by its low positive predictive value. Nearly $75 \%$ of patients with troponin $\mathrm{T}$ concentrations $>0.1 \mu \mathrm{g} / \mathrm{l}$ remained event-free during the first year after presentation. Thus, management programmes based on troponin assays in patients with non-ST elevation coronary syndromes would result in many relatively low risk patients receiving aggressive treatment strategies unless other predictors of risk are also taken into account. This is reflected in the recent FRISC II (fragmin and fast revascularization during instability in coronary artery disease) study, in which an invasive strategy did not significantly reduce death and non-fatal myocardial infarction in the troponin positive subgroup. ${ }^{19}$

\section{Myocardial ischaemia}

It is well established that clinical and electrocardiographic evidence of uncontrolled ischaemia in non-ST elevation coronary syndromes identifies patients at risk of future events $^{20} 21$ and this was confirmed in the present study. Thus, chest pain that failed to settle within 12 hours of admission and Holter ST elevation increased substantially the risk of ischaemic events in the first 30 days, while Holter ST elevation or depression was independently predictive of events at 12 months. $\mathrm{T}$ wave inversion on the presenting ECG was also predictive of events at 12 months but, like other investigators, we found that ST depression on the presenting ECG was not retained as an independent predictor when Holter ST data were included in the multivariate analysis, presumably because of the more prolonged sampling period provided by Holter monitoring. ${ }^{15}$ The relation between uncontrolled ischaemia and future events has led to calls for an ischaemia driven approach to invasive management, an approach validated by the findings of the FRISC II investigators. ${ }^{19}$ Nevertheless, our predictive accuracy data showed that $\geqslant 79 \%$ of patients with clinical or electrocardiographic predictors of cardiac death or non-fatal myocardial infarction remained event-free at least 12 months after presentation, demonstrating important limitations of this approach.

\section{Heart rate variability}

Holter monitoring not only documented ambulatory ST change but also permitted analysis of heart rate variability, which further refined the assessment of risk. Heart rate variability, a convenient measure of autonomic function, is often deranged after myocardial infarction and the severity of the derangement is predictive of outcome. ${ }^{172}$ Similar findings have been reported for patients with unstable angina when prognostic correlates for in-hospital events are additional to those of ST analysis. ${ }^{23}$ Our own data have extended these observations by showing that reductions in time domain indices of heart rate variability early after presentation are associ- ated with a significant increase in rates of cardiac death and non-fatal myocardial infarction during the first 12 months. The association is independent of Holter ST change, as well as of clinical, electrocardiographic, and biochemical markers.

\section{Holter monitoring}

While presenting ECG changes and biochemical markers of myocardial necrosis are well established and routinely used for risk stratification, Holter monitoring is not. With recent advances in recording technology and data analysis, however, its application in the coronary care unit has become increasingly feasible and there is no doubt that continuous ECG monitoring for ST segment shift and heart rate variability may become as routine as that for heart rhythm is currently. This study has confirmed that both ST shift and measures of heart rate variability provide important, independent prognostic information that can be obtained early after presentation to anticipate the period of greatest risk and in this respect Holter monitoring may have advantages over stress testing. The aim of the recently described TIMI (thrombolysis in myocardial infarction) risk score ${ }^{24}$ is to identify, as simply as possible, using readily available information, patients with unstable angina or non-ST elevation myocardial infarction at high risk of further cardiac events. In the absence of continuous ECG monitoring, however, the highest risk group identified (comprising $3.4 \%$ of the sample) has a positive predictive value of only $20 \%$.

\section{Conclusion}

Our findings have emphasised the value of a cumulative approach to risk stratification in non-ST elevation coronary syndromes-most markers of risk have a low level of predictive accuracy when applied independently. Thus, derivation of a risk score from clinical data, troponin $\mathrm{T}$ concentrations, ST monitoring, and heart rate variability was powerfully predictive of short and longer term outcome, successfully identifying a subgroup of patients whose risk of cardiac death or non-fatal myocardial infarction approached 50\% during the first 12 months. This high positive predictive accuracy based on readily available technology exceeds previously reported levels and has the potential to maximise the cost effectiveness of interventional strategies in patients with non-ST elevation coronary syndromes.

\section{Authors' affiliations}

S Kennon, P G Mills, A D Timmis, Department of Cardiology, Barts and the London NHS Trust, London, UK

C P Price, H Clarke, Department of Clinical Biochemistry, Barts and the London NHS Trust, London, UK

P K MacCallum, J Cooper, MRC Epidemiology and Medical Care Unit Charterhouse Square, London, UK

J Hooper, Department of Clinical Biochemistry, Royal Brompton Hospital, London, UK

\section{REFERENCES}

1 Yusuf S, Flather M, Pogue J, et al. Variations between countries in invasive cardiac procedures and outcomes in patients with suspected unstable angina or myocardial infarction without initial ST elevation. Lancet 1998;352:507-14.

2 The TIMI IIIB Investigators. Effects of tissue plasminogen activator and a comparison of early invasive and conservative strategies in unstable angina and non-Q-wave myocardial infarction. Circulation 1994:89:1545-56

3 The VANQWISH Trial Investigators. Outcomes of patients with acute non-Q-wave myocardial infarction randomly assigned to an invasive as compared with a conservative strategy. N Engl J Med 1998;338: 1785-92.

4 Murphy JJ, Connell PA, Hampton JR. Predictors of risk in patients with unstable angina admitted to a district general hospital. Br Heart $J$ 1992;67:395-401.

5 Ayres SM. The electrocardiogram in the acute coronary syndromes. J Am Coll Cardiol 1990; 16:1026-8.

6 Nyman I, Areskog M, Areskog NH, et al. Very early risk stratification by electrocardiogram at rest in men with suspected unstable coronary heart disease. The RISC study group. J Intern Med 1993;234:293-301. 
7 The TIMI III Registry ECG Ancillary Study Investigators. The electrocardiogram predicts one year outcome of patients with unstable angina and non-Q-wave myocardial infarction. J Am Coll Cardiol 1997; 30:133-40.

8 The TRIM investigators. Admission standard electrocardiogram for early risk stratification in patients with unstable coronary artery disease not eligible for acute revascularization therapy. Am Heart $J$ 1999; 137:24-33.

9 Ravkilde J, Nissen H, Horder M, et al. Independent prognostic value of serum creatine kinase isoenzyme MB mass, cardiac troponin T and myosin light chain levels in suspected acute myocardial infarction. J Am Coll Cardiol 1995;25:574-81.

10 The TRIM Study Group Investigators. Applicability of cardiac troponin $T$ and I for early risk stratification in unstable coronary artery disease. Circulation 1997:96:2578-85.

11 Liuzzo G, Biasucci LM, Gallimore JR, et al. The prognostic value of $C$-reactive protein and serum amyloid $A$ protein in severe unstable angina. NEngl J Med 1994;331:417-24.

12 The TRIM Study Group. Very early risk stratification using combined ECG and biochemical assessment in patients with unstable coronary artery disease. Circulation 1998;98:2004-9.

13 The FRISK Study Group. Risk stratification in unstable coronary artery disease. Additive value of troponin T and pre-discharge exercise tests. Eur Heart J 1997; 18:762-70.

14 Morrow DA, Rifai N, Antman E, et al. C-Reactive protein is a potent predictor of mortality independently of and in combination with troponin T in acute coronary syndromes: A TIMI 11 A substudy. J Am Coll Cardiol 1998;31:1460-5.

15 The TRIM Study Group. Admission risk assessment by cardiac troponin $\mathrm{T}$ in unstable coronary artery disease: additional prognostic information from continuous ST segment monitoring. J Am Coll Cardiol 1999;33:1519-27.
16 Braunwald E. Unstable angina: a classification. Circulation 1989;80:410-4

17 Vaishnav S, Stevenson R, Marchant B, et al. The relation between heart rate variability early after acute myocardial infarction and long-term mortality. Am J Cardiol 1994;73:653-7.

18 Stevenson R, Ranjadayalan K, Wilkinson P, et al. Assessment of Holter ST monitoring for risk stratification in patients with acute myocardial infarction treated with thrombolysis. Br Heart J 1993;70:233-40.

19 The FRISC II Investigators. Invasive compared with non-invasive treatment in unstable coronary artery disease: FRISC II prospective randomised multicentre study. Lancet 1999;354:708-15.

20 Cannon CP, Thompson B, McCabe CH, et al. Predictors of non-Q-wave acute myocardial infarction in patients with acute ischemic syndromes: an analysis from the thrombolysis in myocardial ischemia (TIMI) trials. Am J Cardiol 1995;75:977-81

21 Gottlieb SO, Weisfeldt ML, Ouyang P, et al. Silent ischemia predicts infarction and death during 2 year follow-up of unstable angina. J Am Coll Cardiol 1987; 10:756-60.

22 Farrell TG, Bashir Y, Cripps T, et al. Risk stratification for arrhythmic events in postinfarction patients based on heart rate variability, ambulatory electrocardiographic variables and the signal-averaged electrocardiogram. J Am Coll Cardiol 1991;18:687-97.

23 Lanzo GA, Pedrotti P, Rebuzzi AG, et al. Usefulness of the addition of heart rate variability to Holter monitoring in predicting in-hospital cardiac events in patients with unstable angina pectoris. Am J Cardiol 1997:80:263-7.

24 Antman EM, Cohen M, Bernink $P$, et al. The TIMI risk score for unstable angina/non-ST elevation MI. A method for prognostication and therapeutic decision making. JAMA 2000;284:835-42.

\section{IMAGES IN CARDIOLOGY}

\section{Pulmonary arteriovenous fistula}

A

3 year old boy was admitted for nvestigation of generalised cyanosis (arterial oxygen saturation to $81 \%$ ), associated with abnormal density on the left upper lung field (upper panel, middle) and normal cardiac examination.

Lung scanning with 99 Tc-albumin showed a large perfusion defect in the same region and presence of radiotracer in the systemic circulation. Chest computed tomography (lower panel, middle) and magnetic resonance imaging (upper panel, right) showed a huge hypervascular mass in the left hemi thorax compatible with pulmonary vascular fistulas (arrows); smaller additional vascular nodules were present in the right lung. The diagnosis of pulmonary vascular fistulas was confirmed by angiography (lower panel, right; arrow).

Following successful lobotomy and embolisation of the right sided fistulae, the child improved significantly and was seen five months later with arterial oxygen saturation to $91 \%$.

C R P de Castro

N M Ikari

F B Jatene

claudiacastro@innovision.com.br
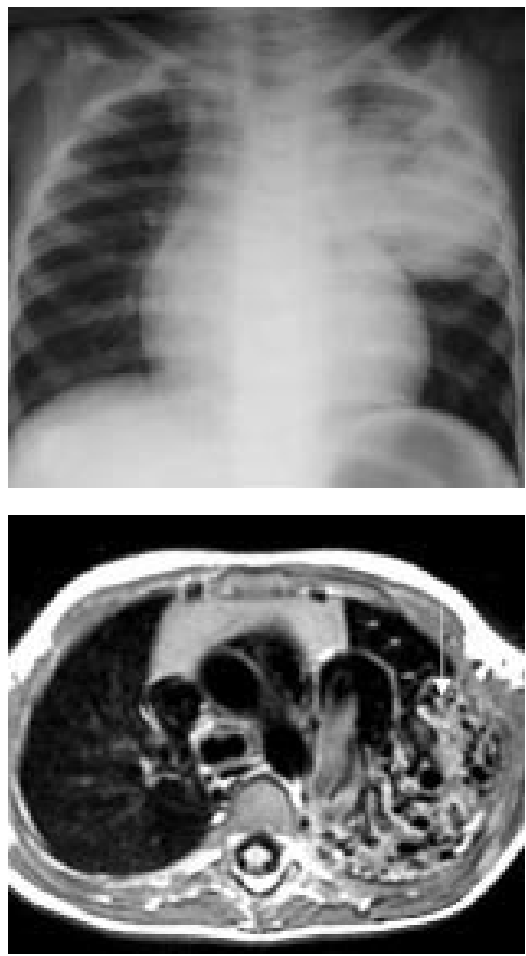
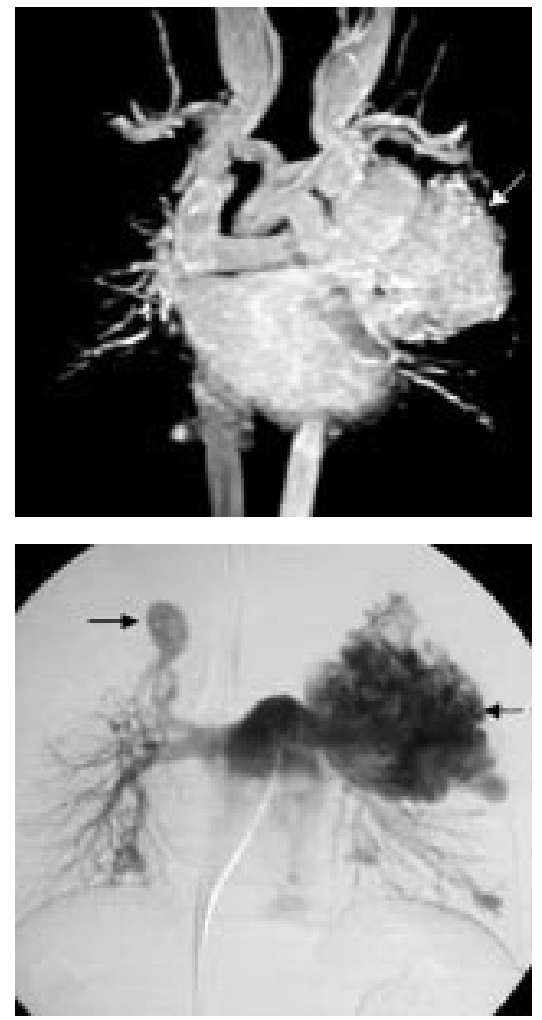